\section{Avoiding the Industrialization of Research Universities: Big and Little Distance Education}

\author{
D. Randy Garrison and Terry D. Anderson
}

\section{Abstract}

\begin{abstract}
The pressure to increase access to higher education while reducing costs raises serious questions with regard to the purpose and goals of the traditional research-intensive university. Moreover, there is considerable rhetoric about reinventing universities based on adoption of communications and learning technologies without clearly defining or articulating educational goals. In contrast to the big industrial model of distance education, an approach to distance education is described that is consistent with the traditional goals and values of creating knowledge through a critical community of learners. This approach, or model, is labeled "little distance education," and its characteristics are defined. Meeting the needs of a new market for continuing professional education available at a distance is also discussed.
\end{abstract}

Some time ago, Wilbur Schramm (1977) described the technologies for instruction as big media and little media. Generally, the intent was to distinguish high-cost, large-audience media from low-cost, small-audience media. Schramm claimed that small or simple media can often provide the necessary conditions needed for a learning task at less cost than large or expensive media. These ideas remain relevant, and it may be useful to revisit them in the context of the challenges that research-intensive universities are facing in the perceived worldwide crisis of access and cost in higher education (Daniel 1997; Dolence and Norris 1995).

A significant change has taken place in higher education during the second half of this century, marked by the collapse of elitism and the opening of universities to large numbers of students. More recently, there has been heightened pressure to increase access to higher education while reducing costs. Many educators, economists, and business leaders have argued that higher education institutions must transform themselves in order to be accessible and cost-efficient. The proposed solution to this "crisis" invariably is the adoption of learning technologies and the substitution of capital for labor (Massy and Zemsky 1996). This focus on access and cost has led to the development of an industrialized model of higher education. The industrialized model is best represented by the emergence of the mega, open universities of the last twenty-five years (Daniel 1997)

Major research-intensive universities must seriously question whether this is the model they should be emulating. The goal of research universities, in addition to their research function, is to enhance the quality of and access to education through communications and learning technologies. The challenge is to achieve this goal in a productive or cost-effective manner. Models for achieving this goal must include affordable and highly interactive technologies. These technologies are not those of the industrialized model.

Evans and Nation (1989) have criticized the dominant open university approach to technology as "instructional industrialism." They suggest that distance education systems evolved from behaviorist models of learning in which course writers have a powerful influence over content and teaching strategies and students are passive receivers of information, "structurally confined to dominated and alienated positions within the distance teaching and learning relationship" (p. 246). Most importantly, discourse is limited and highly regulated in such a way that students are expected to conform and are not allowed to shape their learning experience or challenge the content.

This trend toward the industrialization of higher education and the adoption of mass communication technologies raises serious questions with regard to the purpose and goals of the traditional research-intensive university. Research-intensive universities should begin by clarifying and defending their goals and purposes. This is particularly important with the increased opportunities made possible by developments in information and communications technology and the pressure to adopt distance education approaches in higher education.

The Goals of a University

On the surface, it is ironic that universities, whose raison d'etre is the creation and dissemination of new knowledge, have changed so little in the face of the "knowledge age," an age fueled by enhanced communications capacity. Moreover, communication lies at the heart of higher education. Communication, whether between research collaborators, among and between students and teachers, or between the university and the workplace, is central to both knowledge production and dissemination. 
Brown and Duguid $(1996,13)$ argue that "the core competency of universities is not transferring knowledge but developing it, and that's done within intricate and robust networks and communities."

It i.s particularly striking to see the contrast between the widespread adoptIOn of powerful communications technology within the business ommunity and general population and the tentative and limited adoptIon an se of th.e powers of such technologies for teaching and learning by traditIOnal umversities. While there are many reasons for this trend legitimate and otherwise, it has created tensions within mainstream high er education (Anderson, Varnhagen, and Campbell 1998). There is consi erable rhetoric about adopting communications and learning technologIes, but few concrete action plans that reflect the values and goals of research-intensive universities. Therefore, these plans have little chanc $\sim$ of. b ing implemented and actively supported by faculty.

"Y Ile It IS hyperbole to suggest that higher education is experiencing a cnSIS, transformation is inevitable (Dolence and Norris 1995). Precipit ted by the $\sim$ mergence of powerful new communications technology, hIgher edu atIOn must address the issues of accessibility, cost, and quality of learmng concurrent with the adoption of these technologies. In recent history, technology and cost factors have been allowed to drive this transformation without a clear understanding of desired outcomes.

he issue is o?e of productivity (quality, access, and cost), as opposed to sImply reducIng costs through mass production and delivery. For a research-intensive university, productivity gains must be made first in the area of enhanced quality. If this can be done by increasing access at reduced cost, so much the better.

Major research universities will never be able to compete with the mega, open universities solely on the basis of cost and accessibility-nor should they. Therefore, it is imperative that we understand "what a uni:versity $\sim$ and S,?ould be before getti.ng too excited about technological InnOV.atIOn... . (Paul 1997, 3). ThIS may then lead to the justifiable adoptIon of certaIn forms of communications technology and improved approaches to learning. Effective adoption of innovation only occurs in response to real needs, as defined by the appropriate stake-holders (Rogers 1995).

Paul (1997) states that the goals of a relevant and effective university Include such traditional elements as the search for truth, the creation of knowledge, the preservation of tradition and culture, and the maintenance of a critical perspective. This is certainly true for the research-intensive university. The core competency of the research university is the development of knowledge. Congruent with this idea, teaching and learning should be conceived as an active process of collaboratively constructing knowledge.

The essential means of achieving the traditional goals of the major research university, regardless of the technology, is the provision of sustained reciprocal communication for the purpose of facilitating critical reflection and discourse. It is this process that has been proven effective in facilitating the construction of personal meaning and the continued development of worthwhile knowledge in higher education. All ideas must be open to debate. Educational outcomes should be less about what to think and more about how to think.

The search for truth and the creation of knowledge necessitate the existence of a critical community of learners. It is this collaborative community of learners (scholars and students) that encourages the participants to question existing knowledge and search for new understandings. Critical discourse, supported and enhanced by the advances and opportunities of communications technology, will reveal new approaches to the teaching-learning transaction that can enhance the quality of learning outcomes in higher education by increasing access to critical communities of learners, not simply access to information.

Brown and Duguid (1996) argue strongly that communities of learners are at the core of universities. Learning communities provide a context for active engagement in learning wherein understanding is not achieved by reproducing explicit information without a deeper understanding of the structure of the subject and the important questions for the discipline. According to Brown and Duguid, the real challenge for research-intensive universities is the quality of access provided to its academic communities.

\section{Two Models}

Traditional universities have remained largely unaffected by recent technological developments because they have failed to construct a model of distance education and distributed learning that is consistent with the mandate, culture, and practice of major research universities. The confusion and resistance has come from comparing these research universities to the "mega-universities" (Daniel 1997), which have been driven by technologies consistent with a model that maximizes access and minimizes cost through mass-produced, prescriptive, and largely selfinstructional materials. The model that drives the mega-universities is 
made possible through mass communications technology and remains the dominant model in distance education (Garrison 1997).

The mega-universities emerged as an effective model with which to address the issues of access, cost, and flexibility (Daniel 1997). The result was an industrialized model of teaching and learning. The driving force of the mega-university is the substitution of capital (technology of curriculum production) for labor (the classroom teacher). The ideal is to provide flexibility and control for the learners so they can study when and where they wish. That is, the ideal is to make the learner as independent as possible. This convenience, however, comes at the cost of severely reducing interaction and increasing learner isolation. The emphasis on cost-efficiency for purposes of increased access creates a very different educational experience from that provided by traditional research universities. Along with significant shifts in how we conceive of a quality educational transaction and the emergence of new communications technology, an alternative worldview of distance education is emerging that is more consistent with the culture and practice of research universities (Garrison 1997).

The major research university, with its core mission of creating knowledge, will not be well-served by a mass production and delivery approach to knowledge dissemination. The challenge for research universities is to rethink the nature of the teaching-learning transaction in a distributed environment. We cannot simply weld new communications technologies onto less-than-adequate traditional approaches to teaching and learning. There is, for instance, a tendency to "construe teaching almost entirely in terms of lecturing" (Oblinger and Maruyama 1996). Unfortunately, the lecture has serious limitations with regard to interactivity and establishing a critical community of learners. Therefore, it would not be advisable to build a distributed approach to learning on such a foundation. On the other hand, there is also serious concern with delegating the responsibility for teaching to a Web site constructed with a rigid template that devolves most of the responsibility for learning to the students.

What clearly distinguishes the research university from the mega, open university is its recognition of the centrality of knowledge creation in its teaching and research functions. This is not to protect the status quo, but rather to recognize the core process of the research university with regard to knowledge creation. From the learners' perspective, this knowledge must be acquired in a manner that facilitates deep and meaningful (i.e., quality) learning outcomes. The mass production model of distance education fails to address this core competency of the research university. To address this concern, we propose an alternative model, which we have labeled "little distance education."

\section{Characteristics of Little Distance Education}

Little distance education (LDE) is an emerging model that shares characteristics of both face-to-face instruction and traditional distance education but is built upon the extensive and pervasive networked learning environment. It has many similarities to distributed learning (Oblinger and Maruyama 1996) and is defined by the following characteristics.

LDE Maximizes Interaction. Little distance education systems acknowledge two-way communications as essential to learning. Peer groups are encouraged and collaborative activities are the norm. This usually entails paced systems in which cohort groups proceed through learning sequences. These cohorts allow learning communities to be created and to flourish, thus allowing high levels of interaction without prohibitively high levels of instructional time. The combined use of asynchronous and synchronous communication tools allows a variety of both reflective and spontaneous communications between and among learners and teachers. These tools have the capability of supporting quality interaction potentially superior to both the inhibited discourse found in the face-to-face classroom lecture mode and one-way didactic dialogues common in "big distance education" systems.

LDE Focuses on Meaningful Learning Outcomes. Little distance education focuses on the quality of learning outcomes. The function of materials, teacher guidance, and peer support of the course design serve to motivate, pace, inform, and challenge the student to dig deeply into the subject content and explore the implications of this knowledge with regard to personal and societal constructs. It challenges traditional knowledge as well as personal perspectives and meaning. Since meaning is defined in large part by individuals, the course content and assignments in LDE are sufficiently open-ended to allow for extensive individual exploration, topic selection, and negotiation of course objectives.

LDE Maximizes Active Learning. Little distance education makes extensive use of active learning activities, including simulations, explorations, and explanatory assessment. The costs of creating and distributing these resources are spread among many institutions and 
learners through the use of a network. For example, banks of interactive simulations, learning modules, quizzes, and computer-assisted learning drills are being created and distributed across networks (see, for example, Apple's Education Object Economy at: <http://www.eoe.org>).

These educational resources are commonly written in JavaTM so as to be compatible and distributable across a variety of operating systems. Unlike earlier large-scale computer-assisted learning programs, such as the PLATO experiments, LDE does not focus on the creation of prepackaged, independent study materials so standardized as to become immutable and, therefore, "teacher proof' in the sense that they are not diminished by bad teaching. Rather, LDE uses computer tutorials and simulations that are combined with teacher notes and interactive activities to create complete courses and programs.

LDE Is Flexible in Design. Little distance education courses are revised by every teacher and in every section in which they are taught. Course development consists of defining broad learning goals and outcomes and a set of learning activities that can be modified and supplemented in response to learner or instructor needs and a similar set of evaluation guidelines. LDE can be printed or converted from bytes to matter (in many formats) based on the needs of students or teachers. Storage for LDE does not consist of a warehouse of products, nor does it exist only in the mind or notebooks of the instructor. Rather, course materials are created in hyper-linked and hypermedia format and stored such that they can easily be modified, augmented, annotated, or printed by both instructor and learners as needed. The modular nature of LDE programming creates the opportunity for customization by both teacher and learners to accommodate different learning styles and contexts and the creation and application of new knowledge.

LDE Supports a Systems View. While not dictated by the large-scale efficiencies of big distance education systems, LDE acknowledges the systems within which formal education is developed and delivered. Effective LDE systems provide for learner support services, registration flexibility, credit transfer, accreditation, provision of learning and research resources through electronic delivery and virtual libraries. These courses are embedded within transparent systems so that neither individual faculty members nor individual learners are forced to create ad hoc adaptations of higher education systems.

LDE Is Distributed. The course design of LDE allows the materials and learning activities to be effectively used both at a distance and on campus. Mediated communications supported by a variety of telecommunications technologies are easily exchanged for face-to-face interaction when such interaction is possible and affordable. Similarly, the collaboration, problem solving, and project work of learners can take place within a classroom or be spread across the globe.

LDE Is Compatible with Research Practice. Little distance education shares the tradition and tools associated with the creation of knowledge. Increasingly, networks with collaborative tool-sets are essential to teams of widely distributed researchers. Communications technologies allow for global collaboration and distributed use of scarce source materials and expensive tools. Similarly, these networks are employed in LDE systems in which the focus moves from research to teaching and learning. LDE creates learning environments focused on problem solving, collaborative projects, and exploration of complex environments. Thus, the skills and attitudes acquired through LDE experiences are directly transferable and congruent with the actual work of researchers and other knowledge workers.

LDE Is Cost-Effective. Cost-efficiency cannot be reduced to a simple computation of costs divided by student numbers. McClure (1997) argues that academic productivity is a function of access and learning quality divided by the costs. LDE substantially increases access as courses become available at any time of the day or night and anywhere that dial-up or LAN-based network connectivity is available. Increased accessibility is critical to the survival of publicly funded education. Systems that allow only exclusive and restricted access to their programming will not only fail to meet needs of lifelong learners, but will also find it increasingly difficult to argue for government support when more flexible and accessible private and public sector alternatives are available.

The use of active learning techniques, access to networked resources, customization of content, and support for collaboration all enhance learning above that normally provided in either face-to-face or big distance education settings. Costs are roughly equivalent to face-to-face learning, with capital costs of campus buildings substituted for communications and information technologies. Through increased accessibility and learning effectiveness and constraining costs, productivity in LDE is enhanced without replacing critical labor (knowledge-producing professoriate) with capital. 


\section{The Technologies of Education}

The major research universities are not exempt from issues of accessibility. Even those who argue most emphatically for excellence in research universities recognize the importance of accessibility for qualified students. In reference to the Canadian context, Bercuson, Bothwell, and Granatstein (1997) state that, "major research universities must recognize that their mandate now includes distance education. .." (p. 89) They continue by saying that the primary mission of the major research universities is to teach people how to think, and the challenge is to use the new technologies to enhance this primary mission. Accomplishing this in a distance or distributed learning environment necessitates an understanding of the capabilities of communications technologies that can facilitate educational activities leading to quality learning outcomes.

All forms of distance education are mediated by technology. The technology characteristics of LDE systems are congruent with the needs of the modern research university-namely, a high degree of interactivity, rapid development and deployment of learning products (courses and other learning sequences), and compatible access to the wider research community. Moreover, LDE systems add value to the research university by extending its reach and mandate over temporal and geographical distance. The categorization of a technology as supporting either big or little distance education is rather arbitrary and open to further discussion and change induced by the fluid economics and costs of technology. Nonetheless, we believe that various media lend themselves differentially to application in the face-to-face classroom as well as in little and big distance education systems.

The Technology of Face-to-Face Classrooms. Often the technologies of the face-to-face classroom do not extend beyond blackboards and overhead projectors. The presentation and feedback technologies now appearing in "smart classrooms" enhance the presentation and interaction capacity of the classroom. In addition, they increase the speed and ease with which classroom content can be developed and shared among teachers. Materials developed for presentation in the face-to-face classroom (e.g., PowerPoint slides, streaming video presentations of lectures, etc.) often can be easily adapted for delivery using interactive forms of LDE, thus increasing cost-effectiveness of media applications.

The Technology of Little Distance Education. There are a variety of synchronous and asynchronous communication and presentation tools available today. Synchronous distribution of classroom learning through video and audio teleconferencing was the first medium to be used extensively in LDE systems. However, increasingly LDE systems are making use of the asynchronous capabilities of the Internet-especially the display, hyper-linking, and hypermedia capacity of the World Wide Web (WWW). The early support and development of the Internet by researchintensive universities and the opportunities provided to them through recently announced high-speed research initiatives such as Internet2 and CaNet3 present ideal conditions under which to quickly expand their LDE opportunities. The selection and development of learning materials that use appropriate technologies will insure higher levels of compatibility with the core values and culture of the research-intensive university.

A significant subset of WWW technology is computer conferencing, which has the potential to be, and is rapidly evolving into, the most widely used medium in LDE systems (Harasim et al. 1995). Its popularity is due to the compatibility, power, and access provisions of this mostly text-based medium. Asynchronous computer conferencing supports high levels of interactivity, "anytime, anywhere," while allowing and supporting the type of reflective and substantiated discourse that is highly valued in the research-intensive university. Recorded and broadcast audio is also a technology with potential for application within LDE systems. Audio recording offers very low cost of production, relatively low cost of distribution (especially over the Internet), compatibility with the lecture-based tradition of the research university, and relative ease and inexpensive recording and editing.

The Technology of Big Distance Education. The cluster of technologies most appropriate for big distance education systems include the broadcast technology of television, the large-scale production of correspondence materials in print format, and the development of computer-assisted instructional and multimedia courses such as those developed by PLATO, Academic Systems, and others. The high production costs, coupled with the lack of interactivity inherent in broadcast television, have precluded its adoption by all but the largest of distance education systems, such as the British Open University and the China Television University. Attempts by single universities or consortia to create telecourses have had limited success at the college level, but have been markedly less successful within the research university. The cost of production of quality materials that use any of the media in this grouping is very high and generally requires the industrialized production skills of designers, technicians, and producers, in addition to subject matter 
experts or teachers. These costs can only be recovered through massification and large student numbers that, in turn, must be supported by large and complex administration and support infrastructures.

The big media described previously can be used as a supplementary resource for LDE systems. In such applications, the cost of production and distribution is normally left to publishers and mass media distributors. For example, LDE systems may purchase textbooks, videotapes, or computer-assisted learning sequences, but they will rarely, if ever, enter into the business of producing, publishing, and marketing products for the mass market.

\section{A Learning Community}

The essence of the research university is reflected in the ideal of a critical community of learners. We argue that a crucial component to acquiring meaningful and worthwhile knowledge is a critical community of scholars and learners. It is interesting to note that the more we use the powerful asynchronous technologies such as computer conferencing, the more students feel a need for synchronous communication (Harasim et al. 1995; Fabro 1996). Asynchronous written communication has very different qualities and characteristics from synchronous verbal communication (Kaye 1987; Garrison 1997).

It is becoming clear in distance education that we do not have to abandon the social context as we adopt mediated forms of communication for educational purposes. While there will always be a place for intensive residential experiences, it is possible to create a community of learners in a technologically distributed format. The key element in designing these technologically mediated learning experiences are the educational ideals we are striving to achieve. Within practical limits, educational ideals and learning outcomes must determine the appropriate communications technology. The ideals of the traditional research university should not be abandoned to the driving force of technology. Instead, these ideals should shape the adoption of communications technology and be reflected in the approach to distance education.

Didactic, one-way delivery models of education, whether face-to-face or virtual, are becoming unacceptable to the growing percentage of mature part-time learners enrolled in universities. Add-on approaches such as television broadcast and linking of lecture rooms via video conferencing will inevitably be short-lived and not address the challenges of access concurrent with a quality learning experience. The lasting effect of new communications technology lies in its ability to enhance the quality of the educational transaction consistent with the legitimate needs and ethos of research-intensive universities while improving access. For the working professional, the combination of a quality learning experience accessible both synchronously and asynchronously is a necessity. This will require new approaches to facilitating the teaching-learning transaction. These new approaches will recognize and support critical reflection and discourse within a community of learners, whether it be face-to-face or virtual.

\section{New Markets}

Remaining true to the traditional goals of the research university does not mean ignoring the changing client needs and demographics of higher education. It would be detrimental to the research university to ignore the growing market for post-degree continuing professional education. Particular attention should be given to approaches that meet the needs of the continuing part-time learner. The research university must adopt technologies that will serve full and part-time traditional learners as well as the mature post-degree continuing learner. Cost-recovery models need to be introduced. Major research-intensive universities must become multifaceted if they are to remain relevant in this postmodern age.

Meeting the needs of the nontraditional learner does not mean abandoning the quality standards of the research university. It does mean, however, that new approaches must be adopted that recognize different outcome standards. For the professional with considerable working knowledge, it is no longer acceptable to focus exclusively on abstract theory. The challenge of post-degree professional programs is to translate theory into practice in a multidisciplinary context. The teaching-learning transaction must facilitate the understanding of relevant theoretical knowledge from personal and contextual perspectives. Moreover, knowledge is not the exclusive domain of the university. Experienced professionals possess enormously powerful working knowledge that should be shared with fellow learners. Finally, a quality learning experience for the post-degree professional must be timely. That is, it must have meaning, practical importance, and relevance to the current needs of the learner. For the continuing professional learner, this will reflect a shift in what is currently considered a quality educational expenence. 
Recognizing that a quality educational experience must meet the needs of the "client" is a particular challenge within the major research university. Traditional academicians are hard-pressed to understand the challenges of translating theory to practice and to recognize the practical knowledge of working professionals. This means that innovative and accessible professional part-time graduate programs will likely experience considerable resistance during the approval process. This is made worse by the need to mount these programs in a timely manner. To respond to emerging market opportunities, programs must be designed and approved in short periods of time. New standards and approval procedures will likely have to be implemented, or at least attitudes will have to change in order to expedite the realization of market-driven professional programs.

Another key characteristic of a continuing professional education program is accessibility to information and human knowledge sources. It is not sufficient to simply provide access to information. A quality learning experience demands that students have opportunities to interact with the professor and fellow learners in a sustained manner. These programs represent an opportunity to understand and utilize the full capabilities of communications technology. The challenge is to make it central to the educational transaction and not simply an extension of what is already done or an enhancement of less-than-adequate conventional processes. In addition, it is not acceptable to rely on one technology. Research universities have much to learn with regard to making quality learning experiences accessible off-campus or in a virtual environment. At a minimum, a combination of face-to-face and mediated, as well as synchronous and asynchronous communication options must be employed to provide a full quality learning experience.

Quality learning experiences in higher education require a flexible mix of collaborative synchronous communication and reflective asynchronous formats. Students must be assured a quality learning experience defined in terms of relevant content combined with sustained opportunities for critical reflection and discourse. The challenge of a quality program is to create a design that addresses relevant content and its practical application, is accessible to working professionals, and can be completed in a reasonable period of time (two to three years).

One powerful strategy is to adopt a cohort-based approach to learning. Furthermore, establishing a climate for critical discourse is facilitated considerably by bringing the cohort together for a three-week face-toface institute at the start of each academic year. Initially, this would allow time for students to get to know each other as well as be introduced to the technical intricacies of the communications technology. Subsequently, course content best suited for a face-to-face context could be addressed during such an institute.

Without question, research universities must recognize the growing market and influence of the post-degree continuous learner. Addressing the needs of this market will also have secondary beneficial influences in changing for the better traditional on-campus approaches to teaching and learning. For its own viability, the research-intensive university must continue to serve its students as well as its corporate and nonprofit clients and benefactors. The best way to do this in a quality and personalized manner is through the adoption of little distance education communications technology.

\section{Conclusion}

Strategies to incorporate communications technology must not compromise the ideals and standards of excellence traditionally found in the major research-intensive universities. While accessibility and cost are challenges research universities must face, they are not defining characteristics as they are with the mega, open universities. The industrialization (massification) of the research university should be resisted. Research universities must find a balance between the traditional low-access elitism of the research-intensive universities and the "massification" of the mega, open universities. Almost by definition the major research universities are elitist in the sense that they attempt to attract the best and brightest students and faculty. Elitism, however, must not be maintained on grounds other than those of competence and ability. It is not in the best interest of the research university to compete with the mega universities in being open to the mass market. This would be a serious threat to the quality of the research and teaching of traditional universities. It would also diminish the prestige of a degree from a major research university.

It is without apology that we say that, in the general trend toward the massification of higher education, the major research universities must distinguish themselves by basing access on intellectual merit. T e research university cannot succumb to the politically correct democratIc ideal of equal access regardless of talent. The colleges and mega, open universities should address these legitimate concerns with regard to access and the demand for mass higher education. Not all students want 
or will benefit from an education that integrates teaching and research. Industrialization and massification run counter to critical exploration and creation of new knowledge. For research-intensive universities, the adoption of distance education communication technologies should enhance the quality of the learning experience while increasing the convenience of access. In this way, productivity gains can be made.

Despite the rhetoric surrounding developments in technologies for distance education and the imperative to adopt these learning technologies, most traditional research universities remain largely unaffected by such developments. Elite research universities have failed to define and adopt a model of distance education that is consistent with their mandate, culture, and practice. Moreover, the real threat is not the mega-universities or the "for-profit" institutions, such as the University of Phoenix, but accessible and relevant programs from other renowned research universities.

While the focus of this paper has been on research-intensive universities, other institutions could also benefit from "little distance education." Certainly teaching universities and colleges could enhance learning through the application of LDE technologies. However, all institutions of higher education that have the teaching-learning transaction as a defining characteristic can benefit from LDE educational technologies with the capability to bring together a critical community of learners and the flexibility to meet emerging learning needs with the express purpose of enhancing the quality of learning outcomes.

We believe that there is a model of distance education that can preserve and enhance the quality of the teaching-learning transaction while increasing access to education. At the core of this model is the maintenance of quality of content, delivery, and students. The model advocated here is the creative adoption of systems based on "little distance education" technologies that are not only affordable over time but, most importantly, support sustained two-way critical discourse and the realization of a virtual community of learners. In this way, the quality standards of the research-intensive university can be maintained while increasing access to all qualified learners.

\section{References}

Anderson, T., S. Varnhagen, and K. Campbell. 1998. Faculty adoption of teaching and learning technologies: Contrasting earlier adopters and mainstream faculty. Canadian Journal of Higher Education 28 (2-3): 71-98.
Bercuson, D., R. Bothwell, and J. L. Granatstein. 1997. Petrified campus: The crisis in Canada's universities. Toronto: Random House of Canada.

Brown, J., and P. Duguid. 1996. Universities in the digital age. Change 28 (4): $10-19$.

Daniel, J. S. 1997. Why universities need technology strategies. Change 29 (4): 11-17.

Dolence, M., and D. Norris. 1995. Transforming higher education: A vision for learning in the 21st century. Ann Arbor, MI: Society for College and University Planning.

Evans, T., and D. Nation. 1989. Critical reflections on distance education. London: Falmer Press.

Fabro, K. 1996. Computer conferencing in higher education: An exploration of communication and cognitive development issues. Master's thesis, University of Calgary, Canada.

Garrison, D. R. 1997. Computer conferencing: The post-industrial age of distance education. Open Learning 12 (2): 3-11.

Harasim, L., S. R. Hiltz, L. Telus, and M. Turoff. 1995. Learning networks: A field guide to teaching and learning online. Cambridge, MA: MIT Press.

Kaye, T. 1987. Introducing computer mediated communication into a distance education system. Canadian Journal of Educational Communication 16:153-166.

Massy, W., and R. Zemsky. 1996. Information technology and academic productivity. Educom Review 31 (I): 12-15.

McClure, P. 1997. Technology in university teaching and learning: Benefits and barriers from a technology viewpoint. Online Computer Library Centre. Available online at: <http://www.oclc.org/oclc/ man/1 0045rld/mcclure.htm>.

Oblinger, D., and M. Maruyama. 1996. Distributed learning. CAUSE Professional Paper Series, no. 14. Boulder, CO: CAUSE.

Paul, R. 1997. The university of the future: Learning from 15 years of distance education. Paper presented at 18th ICDE World Conference, 2-6 June, The Pennsylvania State University, University Park, PA.

Rogers, E. 1995. Diffusion of innovations. 4th ed. New York: Simon \& Shuster.

Schramm, W. 1977. Big media, little media: Tools and technologies for instruction. London: Sage. 